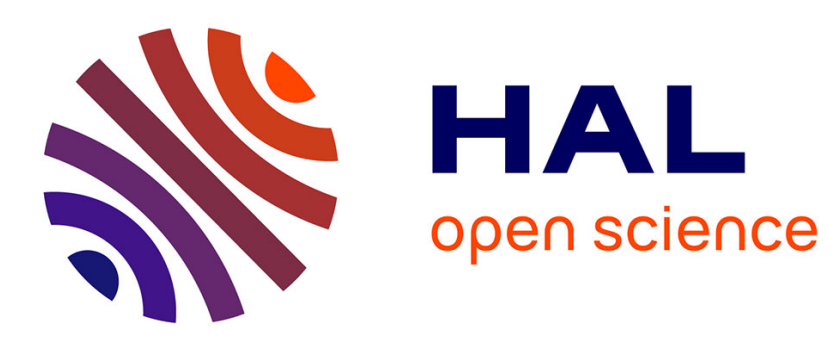

\title{
Nouvelle méthode de profilométrie capacitive des porteurs libres et des centres profonds dans les semiconducteurs
}

B. Remaki, B. Balland

\section{- To cite this version:}

B. Remaki, B. Balland. Nouvelle méthode de profilométrie capacitive des porteurs libres et des centres profonds dans les semiconducteurs. Revue de Physique Appliquée, 1987, 22 (11), pp.13811388. 10.1051/rphysap:0198700220110138100 . jpa-00245688

\section{HAL Id: jpa-00245688 https://hal.science/jpa-00245688}

Submitted on 1 Jan 1987

HAL is a multi-disciplinary open access archive for the deposit and dissemination of scientific research documents, whether they are published or not. The documents may come from teaching and research institutions in France or abroad, or from public or private research centers.
L'archive ouverte pluridisciplinaire HAL, est destinée au dépôt et à la diffusion de documents scientifiques de niveau recherche, publiés ou non, émanant des établissements d'enseignement et de recherche français ou étrangers, des laboratoires publics ou privés. 


\title{
Nouvelle méthode de profilométrie capacitive des porteurs libres et des centres profonds dans les semiconducteurs
}

\author{
B. Remaki et B. Balland \\ Laboratoire de Physique de la Matière (LA C.N.R.S. 358), I.N.S.A. de Lyon (Bât. 502), 20 avenue Albert \\ Einstein, 69621 Villeurbanne Cedex, France
}

(Reçu le 20 novembre 1986, révisé le 10 juillet 1987, accepté le 20 juillet 1987)

\begin{abstract}
Résumé. - Nous proposons une nouvelle méthode de profilométrie capacitive des impuretés dans les matériaux semiconducteurs, adaptée aux cas des fortes concentrations de pièges. La procédure expérimentale repose sur la mesure de la caractéristique $C$ - $V$ haute fréquence en régime hors équilibre, à l'aide d'un dispositif corrélateur rapide. L'évolution de la charge d'espace étant contrôlée uniquement par les porteurs libres, la détermination de leur profil de concentration n'est pas faussée par la réponse d'un ou plusieurs niveaux profonds. De plus, la mesure de la caractéristique $C-V$ haute fréquence en situation d'équilibre, et l'élaboration d'une expression analytique nouvelle formulant la largeur de la zone de transition, donnent accès au profil des centres profonds. Après avoir défini le domaine de validité de notre méthode, nous l'avons appliquée dans le cas du silicium monocristallin ayant subi soit une diffusion d'or, soit une implantation ionique de phosphore.
\end{abstract}

\begin{abstract}
A new method is described for the determination of impurity profiles in semiconductors. The technique is based on measurement of high frequency $C-V$ characteristics under nonequilibrium conditions, using a fast correlator apparatus. The space charge is controlled only by the free carriers. Their spatial distribution can be obtained for a wide range of conditions, specifically for the case of very high trap concentration and abrupt changes of the spatial distributions of either or both of the trap centres. This method is completed by measurement of classical at-equilibrium high frequency $C-V$ characteristics and a new well adapted analytical formulation of the edge region allow also to get trap concentration profile. After a detailed survey of its limitations, our method is tested with gold-diffused and implanted phosphorus.
\end{abstract}

\section{Introduction.}

L'étude des propriétés électriques et électro-optiques des matériaux semiconducteurs nécessite de connaître la concentration des porteurs libres à l'équilibre thermodynamique (c'est-à-dire le dopage apparent), et des pièges à majoritaires et/ou à minoritaires. Pour ce faire, plusieurs techniques d'analyse sont envisageables. Citons parmi les plus importantes, la spectroscopie de masse (S.I.M.S. : Secondary Ion Mass Spectroscopy), les techniques de réaction nucléaires, la résistivité et la capacité différentielle, dite technique $C-V$. Elles ne fournissent pas toutes, les mêmes informations physiques. Par exemple, la méthode S.I.M.S. donne le nombre d'impuretés physiquement présentes dans le semiconducteur, indépendamment de leur état de charge et de leur position (substitutionnelle ou interstitielle) dans le réseau cristallin; par contre, des méthodes électriques comme la résistivité ou la capacité ne renseignent que sur le nombre de celles qui sont électriquement actives. Les résultats ne sont identiques, pour une même impureté (additif de dopage) que si son activité électrique est de $100 \%$. De plus, les sensibilités ne sont pas comparables; par exemple, $10^{15}$ atomes. $\mathrm{cm}^{-3}$ en limite inférieure pour l'analyse S.I.M.S., et $10^{17}$ atomes. $\mathrm{cm}^{-3}$ en limite supérieure pour la méthode capacitive ; cette valeur est liée à la faible extension de la zone de charge d'espace pour les fortes concentrations des impuretés de dopage.

Méthode simple et non destructive pour le dispositif-test, la technique $C(V)$ apparaît bien adaptée pour des études profilométriques dans les matériaux semiconducteurs, en raison de : i) la facilité de mise en œuvre, ii) la sensibilité, iii) la possibilité de mesures in situ. Aussi est-elle fréquemment utilisée pour déterminer les profils de concentration des 
porteurs libres et des impuretés électriquement actives. Elle constitue un puissant outil d'analyse ; notons qu'une part importante d'un colloque relatif à l'étude des additifs de dopage lui a été consacrée [1].

Cette technique repose sur l'exploitation de la caractéristique $C(V)$ d'une jonction Schottky ou PN abrupte et dissymétrique, polarisée en inverse $[2,3]$. Utilisée en association avec une technique de spectroscopie transitoire telle que la D.L.T.S. (Deep Level Transient Spectroscopy) [4], elle permet d'obtenir le profil de concentration des additifs de dopage (impuretés «superficielles ») et des pièges (défauts et impuretés "profondes") lorsque ces derniers sont très peu nombreux vis-à-vis des porteurs libres [5-8]. Dans le cas contraire, les résultats expérimentaux sont d'interprétation difficile; la concentration des porteurs n'est alors déterminée qu'avec une grande incertitude (forte distorsion des profils) $[9,10]$. Certes, un progrès sensible a été réalisé dans ce dernier cas par l'utilisation de la C.C.-D.L.T.S. (D.L.T.S. à capacité constante) [1114] et de la capacité basse fréquence [13]. Mais, deux restrictions importantes en limitent la potentialité :

i) La mesure de la capacité basse fréquence est en général peu précise, notamment pour les structures à fort courant inverse.

ii) La C.C.-D.L.T.S. nécessite la mise en œuvre d'un asservissement délicat qui peut réduire considérablement la sensibilité de la mesure [14].

Nous présentons ici, une nouvelle méthode capacitive qui permet de déterminer correctement la concentration des porteurs libres, en éliminant toute perturbation sur cette mesure, due à la présence des centres profonds. Pour ce faire, nous exploitons le comportement des porteurs libres et le phénomène de "gel des pièges » dans un état d'occupation préalablement choisi. Le principe est le suivant :

i) La mesure de la capacité haute fréquence en régime hors équilibre (notée $C_{\mathrm{he}}(V)$ ) est réalisée à l'aide d'un dispositif corrélateur. Dans cette expérience, l'évolution de la charge d'espace est contrôlée uniquement par les porteurs libres dont le profil de concentration peut ainsi, être obtenu indépendamment de toute contribution des pièges.

ii) Grâce à une expression analytique que nous avons établie, nous déterminons la largeur de la zone de transition $\lambda$ à partir d'une mesure.

iii) L'exploitation de la caractéristique haute fréquence en régime d'équilibre thermodynamique $C_{\mathrm{hf}}(V)$ et des données précédentes permet d'obtenir le profil des centres profonds.

Notons que l'idée d'exploiter la situation de « gel des pièges " a déjà été utilisée par Brotherton [15] en opérant à très basse température. Nous en avons revu les fondements en améliorant et en complétant :

i) l'aspect expérimental par la mesure de la capa- cité instantanée corrélée à une polarisation inverse «fugitive». Ce qui permet d'élargir le domaine d'utilisation en température et en profondeur énergétique des pièges.

ii) l'aspect théorique par une exploitation plus adaptée et plus complète.

Nous décrivons, dans ce qui suit, le dispositif expérimental et les modèles analytiques d'exploitation. Après avoir précisé les limites d'utilisation de notre méthode, nous commentons les résultats obtenus pour les profils d'impuretés diffusées et implantées dans le cas où la concentration en centres profonds excède celle des porteurs libres.

\section{Procédure expérimentale.}

Pour simplifier, nous ne considérons que le cas d'une diode Schottky/ $N$ contenant des pièges à électrons (à majoritaires), en concentration $N_{t}$, à un niveau de profondeur énergétique $E_{\mathrm{t}}$. La constante (ou « vitesse » en $\mathrm{s}^{-1}$ ) d'émission, $e_{\mathrm{n}}$ et le coefficient (ou "vitesse », en $\mathrm{cm}^{-3} \cdot \mathrm{s}^{-1}$ ) de capture de ces pièges peuvent être reliés l'un à l'autre en utilisant les conditions d'équilibre. En appliquant le principe du bilan détaillé on obtient :

$$
\begin{aligned}
e_{\mathrm{n}}=c_{\mathrm{n}} \cdot n(x) \cdot \exp \left\{\left(E_{\mathrm{t}}-E_{\mathrm{f}}\right) / k T\right\}= \\
=K_{\mathrm{n}} T^{2} \sigma_{\mathrm{n}} g \exp [-\Delta E / k T]
\end{aligned}
$$

en notant par

$n(x)$, la concentration des porteurs libres à la profondeur $x$, dans les conditions d'équilibre thermodynamique ;

$\sigma_{n}$, la section efficace $\left(\mathrm{cm}^{2}\right)$ de capture thermique du niveau-piège à l'égard des électrons ;

$g$, un facteur de dégénérescence du défaut ;

$K_{\mathrm{n}}$, un facteur constant (soit $1,065 \times 10^{21}$ $\mathrm{K}^{-2} \cdot \mathrm{s}^{-1} \cdot \mathrm{cm}^{-2}$ dans le cas de $\left.\mathrm{Si}\right)$;

$\Delta E=\left(E_{\mathrm{c}}-E_{\mathrm{t}}\right)$ est l'énergie d'activation du niveau-piège relativement à la bande de conduction ; $q$, charge élémentaire $\left(q=1,6 \times 10^{-19}\right.$ coulombs) ;

$k$ : constante de Boltzman $\left(k=8,62 \times 10^{-5}\right.$ $\mathrm{eV} \cdot \mathrm{K}^{-1}$ ).

2.1 DisPositif EXPÉRIMENTAL. - La figure 1a donne le schéma synoptique du dispositif réalisé. La mesure de la capacité (haute fréquence) est effectuée avec un capacimètre Boonton 72B travaillant à $1 \mathrm{MHz}$. La structure étudiée est soumise alternativement aux tensions $V_{\mathrm{d}}$ et $V_{\mathrm{r}}$ à travers une porte analogique (LF 11333) commandée par un générateur de signaux rectangulaires (Systron Donner 101C). $V_{\mathrm{d}}$ polarise la diode étudiée légèrement en direct (environ 0,5 V) afin de "rafraîchir » périodiquement le remplissage des pièges, c'est-à-dire de maintenir ces derniers dans l'état « occupé » (configuration décrite sur la Fig. 2a). $V_{r}$ est une rampe 


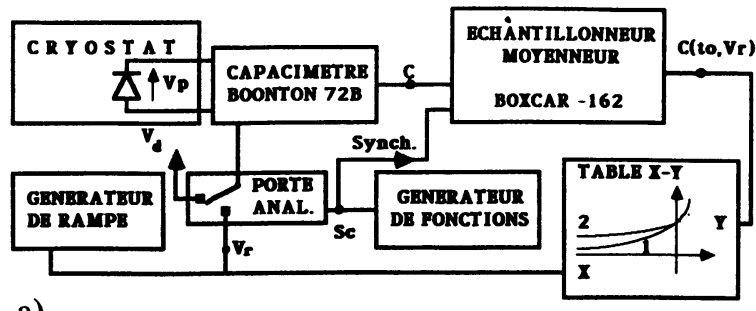

a)

b)

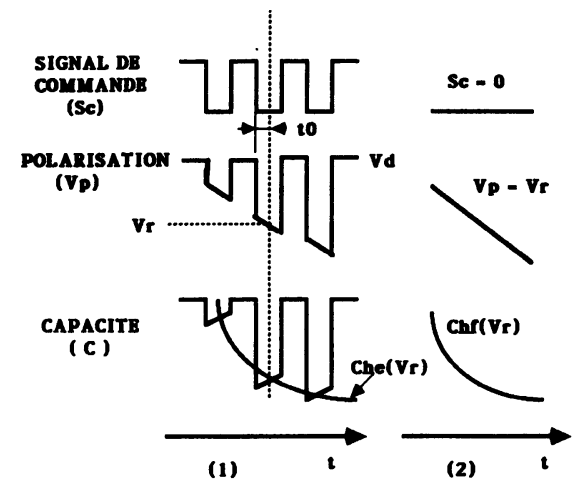

Fig. 1. - Principe de la méthode de mesure : a) schéma du dispositif expérimental permettant l'enregistrement et l'exploitation des courbes quantitatives $C_{\text {he }}\left(V_{\mathrm{r}}\right)$ et $C_{\mathrm{hf}}\left(V_{\mathrm{r}}\right)$; b) évolution temporelle du signal pendant la mesure (la pente de $V_{\mathrm{p}}$ est assez faible pour que la tension $V_{\mathrm{r}}=V_{\mathrm{p}}$ soit considérée comme constante pendant la mesure $C_{\mathrm{he}}\left(V_{\mathrm{r}}\right)$ ), les diagrammes (1) et (2) correspondent respectivement $C_{\text {he }}\left(V_{\mathrm{r}}\right)$ et $C_{\mathrm{hf}}\left(V_{\mathrm{r}}\right)$.

[Principle of the measurement method: a) schematic diagram of $C_{\mathrm{he}}\left(V_{\mathrm{r}}\right)$ and $C_{\mathrm{hf}}\left(V_{\mathrm{r}}\right)$ showing the principle of the measurement method; $b$ ) signal evolution during the measurement (the slope of $V_{\mathrm{p}}$ is small enough so that we can consider $V_{\mathrm{r}}$ as being constant during the capacitive measurement), (1) and (2) diagrams of $C_{\mathrm{he}}\left(V_{\mathrm{r}}\right), C_{\mathrm{hf}}\left(V_{\mathrm{r}}\right)$.]

lente de tension sous laquelle l'échantillonneurmoyenneur (Boxcar 162) fait l'acquisition de la capacité instantanée $C\left(t_{0}, V_{\mathrm{r}}\right)$ correspondant à la zone désertée. La figure $1 \mathrm{~b}$ représente des diagrammes décrivant l'évolution temporelle des divers signaux. Le temps $t_{0}$ qui sépare la mise sous polarisation inverse et la mesure de la capacité instantanée $C\left(t_{0}, V_{\mathrm{r}}\right)$, est choisi très petit comparativement à la constante de temps d'émission, soit $e_{\mathrm{n}}^{-1}$. En présence de plusieurs niveaux-pièges, celle-ci est l'inverse de la «vitesse » d'émission des pièges les plus « rapides » (souvent les moins profonds), soit $e_{\mathrm{n} \cdot \max }$.

Il est évident que si, à une température $T$, le temps $t_{0}$ est faible devant $e_{\mathrm{n} \text { max }}^{-1}, C\left(t_{0}, V_{\mathrm{r}}\right)$ est la capacité hors équilibre thermodynamique :

$$
t_{0}<e_{\mathrm{n} \cdot \max }^{-1} \rightarrow C\left(t_{0}, V_{\mathrm{r}}\right)=C_{\mathrm{he}}\left(V_{\mathrm{r}}\right) .
$$

Dans cette expérience, tous les pièges sont maintenus dans l'état « occupé » pendant la mesure de la capacité $C_{\mathrm{he}}\left(V_{\mathrm{r}}\right)$. La charge d'espace résulte uniquement de l'absence des porteurs libres dans la zone

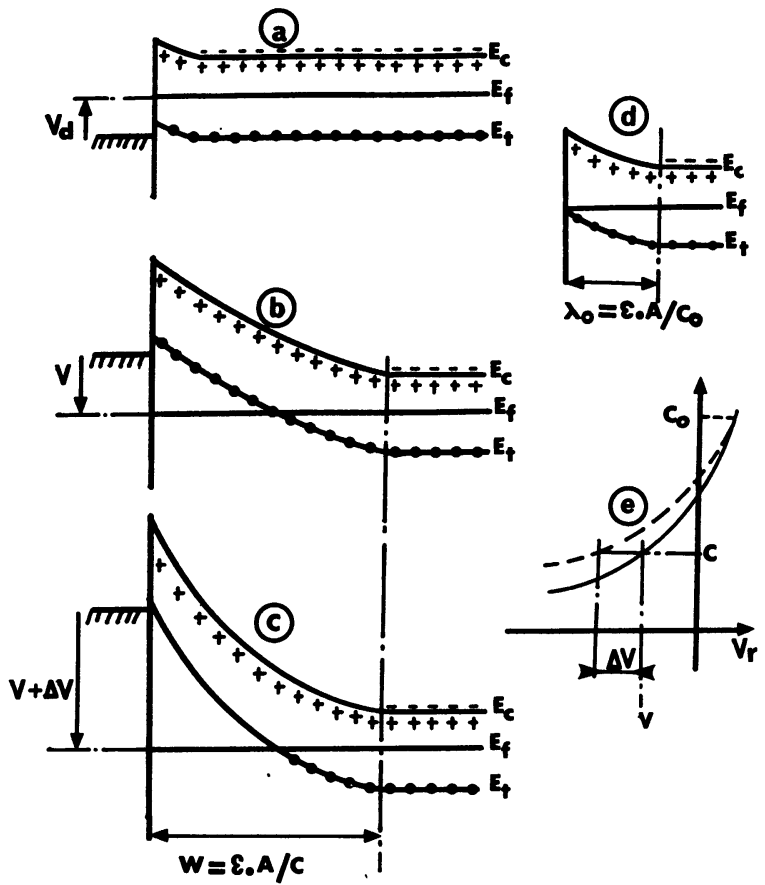

Fig. 2. - Evolution avec la polarisation du diagramme énergétique des bandes et de la configuration de la charge d'espace d'une diode Schottky/ $N$ comportant un niveaupiège : a) diagramme des bandes sous tension de polarisation directe, b) régime hors équilibre sous polarisation inverse, c) régime d'équilibre thermodynamique sous polarisation inverse, d) configuration pour laquelle $\lambda_{0}=w$, e) $C_{\mathrm{he}}\left(V_{\mathrm{r}}\right)$ et $C_{\mathrm{hf}}\left(V_{\mathrm{r}}\right)$ sont les capacités hors équilibre (trait plein) et à l'équilibre (pointillés) en fonction de la polarisation.

[Energy-bans diagram and the space-charge distribution evolution of a Schottky/ $N$ diode with one deep level : a) band diagram forward bias voltage, b) and c) the space charge in the depletion at constant capacitance out equilibrium and at thermal equilibrium respectively, d) space-charges distribution with $\lambda_{0}=w$, e) $C_{\mathrm{he}}\left(V_{\mathrm{r}}\right)$ is the thermal equilibrium capacitance (full line) and $C_{\mathrm{hf}}\left(V_{\mathrm{r}}\right)$ is the non-equilibrium capacitance (dotted line) v.s. bias.]

désertée (Fig. 2b). Leur profil de concentration est alors obtenu, indépendamment de la présence de tout niveau profond, si la condition (2) est satisfaite. Celle-ci peut toujours être réalisée en choisissant la température adéquate. Pour ce faire, on utilise un cryostat pourvu d'un circuit de refroidissement à l'azote liquide.

La rampe de tension $V_{r}$ est choisie suffisamment lente afin de pouvoir tracer directement la caractéristique $C_{\text {he }}\left(V_{\mathrm{r}}\right)$ sur une table $X-Y$ classique. Notons que pour un piège très profond, le régime hors équilibre peut être atteint en opérant à très basse température, sans utiliser de dispositif échantillonneur [15].

La valeur inférieure du temps $t_{0}$ est limitée par le temps de réponse du capacimètre à $1,5 . \mathrm{ms}$. Afin 
d'étendre la gamme d'utilisation du dispositif, nous avons ramené la constante de temps du capacimètre Boonton à $50 \mu$ s par suppression du filtre de sortie ; l'augmentation du niveau de bruit qui en résulte est largement compensée par le moyennage effectué par l'échantillonneur. Cette augmentation de la bande passante du système est très importante car elle permet d'étendre la validité de la méthode aux pièges peu profonds (Fig. $3 b$ ).

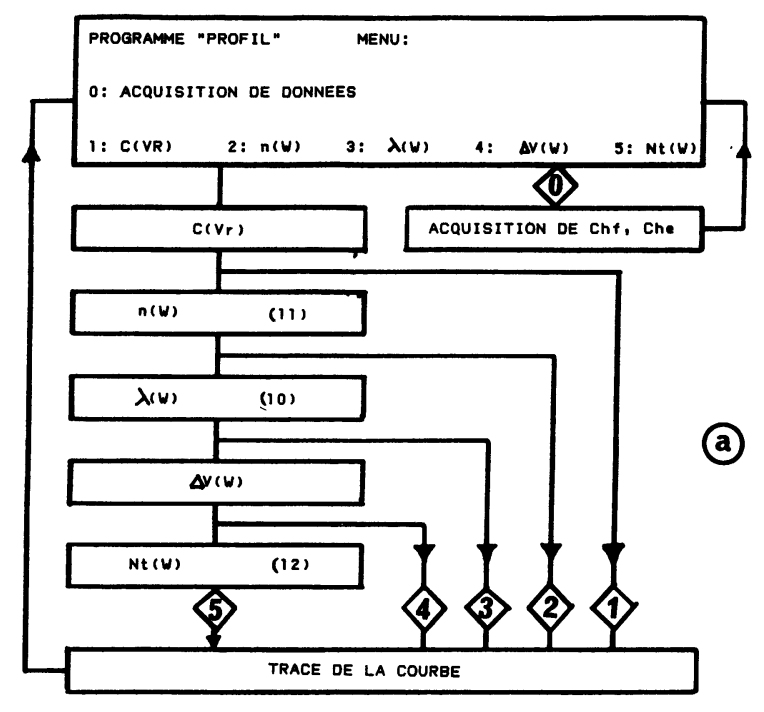

\begin{tabular}{|c|c|}
\hline TEMPERATURE & $77 \cdot 400 \cdot \mathrm{K}$ \\
\hline BANDE PASSANTE & $20 \mathrm{KHz}$ \\
\hline DENSITE DE PORTEURS LIBRES & $10^{11}<\mathrm{n}<10^{17} \mathrm{~cm}^{-3}$ \\
\hline DENSITE D'ACCEPTEURS (*) & $10^{-5}<N t / N d<1$ \\
\hline DENSITE DE DONNEURS (*) & $10^{-5} \mathrm{Nd}<\mathrm{Nt}<10^{17} \mathrm{~cm}^{-3}$ \\
\hline BANDE D'ENERGIE & $0.12<E t<E i \quad(S i)$ \\
\hline
\end{tabular}

Fig. 3. - Organigramme d'acquisition et de traitement des données : a) programme d'acquisition et d'exploitation des données, b) domaine expérimental de validité.

[Data processing block diagram : a) data acquisition and manipulation program, b) limitation of the method.]

La caractéristique haute fréquence à l'équilibre thermodynamique, $C_{\mathrm{hf}}\left(V_{\mathrm{r}}\right)$ est obtenue en polarisant directement la diode par la rampe lente de tension soit $V_{\mathrm{r}}(t)$, la porte analogique est maintenue en position « $V_{\mathrm{r}}$ ", comme indiqué sur le schéma $(S c=0)$ de la figure 1b. Par rapport au régime hors équilibre précédent, la charge d'espace est augmen-' tée d'une quantité correspondant au vidage des pièges (Fig. 2c). L'exploitation des deux caractéristiques $C_{\text {he }}\left(V_{\mathrm{r}}\right)$ et $C_{\mathrm{hf}}\left(V_{\mathrm{r}}\right)$ permet d'accéder au profil de concentration des centres profonds, dans le cas d'un niveau unique ou largement prépondérant.

Cette technique est en principe, applicable au cas des matériaux semiconducteurs comportant plusieurs niveaux profonds. Le profil de chacun d'eux est obtenu en procédant à deux mesures réalisées avec deux valeurs différentes, de $t_{0}:$ l'une très inférieure à la constante de temps d'émission (gel des pièges dans l'état occupé), et au contraire, l'autre est très supérieure (pièges vides). Entre ces deux expériences, la charge d'espace doit varier de la seule contribution du niveau considéré.

En pratique, ces différents régimes hors équilibre thermodynamique sont en général, irréalisables à la même température :

- soit en raison de la très grande dispersion des vitesses d'émission (niveaux de profondeur très différente),

- soit au contraire, pour des problèmes de sélectivité (niveaux très voisins).

De plus l'exploitation analytique est plus complexe (en particulier, le calcul de la largeur de la zone de transition $\lambda$ ), surtout sans connaissance préalable du nombre de niveaux et de leurs caractéristiques cinétiques.

En définitive, pour un matériau comportant plusieurs niveaux profonds, seul le profil de concentration des porteurs libres, $N(x)$ est déterminé. Remarquons qu'en présence de pièges accepteurs, $N(x)$ est évidemment différent du profil des additifs de dopage :

$$
N(x)=N_{\mathrm{d}}(x)-N_{\mathrm{t}}(x)
$$

en notant respectivement par $N_{\mathrm{d}}(x)$ et $N_{\mathrm{t}}(x)$, les concentrations des additifs donneurs superficiels et des centres accepteurs profonds.

\subsection{FORMULATION THÉORIQUE ET EXPLOITATION} DES RÉSULTATS. - La figure 2 représente l'évolution avec les conditions de polarisation, du diagramme des bandes d'énergie et de la configuration de la charge d'espace, pour une diode Schootky/N comportant des pièges à électrons situés énergétiquement en un niveau profond $E_{\mathrm{t}}$. En particulier, les schémas $2 b$ et $2 c$ décrivent respectivement, la situation en régime hors équilibre ou à l'équilibre thermodynamique, sous polarisation inverse et à capacité constante. Dans ces deux cas, l'intégration de l'équation de Poisson dans la zone désertée, conduit à la formulation de la différence de potentiel entre la surface (contact métal-semiconducteur) et le volume du substrat.

i) En situation hors-équilibre thermodynamique, comme l'indique le schéma $2 b$, tous les centres profonds ayant été préalablement remplis sont "gelés", et donc occupés par des électrons. Pour $0<x<w$ la densité volumique de charge est $\rho(x)=+q N(x)$, où $N(x)$ représente le dopage apparent du matériau (centres positifs non compensés électriquement par des électrons libres). 
On obtient donc,

$$
\begin{array}{ll}
\frac{\mathrm{d}^{2} V}{\mathrm{~d} x^{2}}=-\frac{q N(x)}{\varepsilon} & \text { ou encore } \\
& \frac{q N(x)}{\varepsilon}=\frac{\mathrm{d}}{\mathrm{d} x}\left\{V-x \frac{\mathrm{d} V}{\mathrm{~d} x}\right\}
\end{array}
$$

soit

$$
\int_{0}^{w} x \cdot[q N(x) / \varepsilon] \cdot \mathrm{d} x=V(w)-w\left\{\frac{\mathrm{d} V}{\mathrm{~d} x}\right\}_{w}
$$

d'où

$$
V+V_{\mathrm{b}}=(q / \varepsilon) \int_{0}^{w} N(x) \cdot x \cdot \mathrm{d} x
$$

en notant par,

$V_{\mathrm{b}}$, la hauteur de la barrière de potentiel de la jonction,

$V$, la tension de polarisation appliquée extérieurement en régime hors équilibre,

$\varepsilon$, la permittivité diélectrique $(\varepsilon=1,06 \mathrm{pF} / \mathrm{cm}$ pour $\mathrm{Si}$ ),

$w$, la largeur totale de la zone de charge d'espace.

ii) En situation d'équilibre thermodynamique, les centres sont vides (par exemple, ionisés positivement pour des centres donneurs) sur une profondeur spatiale $w-\lambda$. Le même calcul que précédemment conduit à :

$$
\begin{aligned}
V+V_{\mathrm{b}}+\Delta V & =(q / \varepsilon) \int_{0}^{w} N(x) \cdot x \cdot \mathrm{d} x+ \\
& +(q / \varepsilon) \int_{0}^{w-\lambda} N_{\mathrm{t}}(x) \cdot x \cdot \mathrm{d} x .
\end{aligned}
$$

La variation de potentiel $\Delta V$ est ici très simplement mesurée par la différence entre les tensions qui correspondent à la même valeur de $C_{\mathrm{hf}}$ et de $C_{\text {he }}$ (capacité constante); nous évitons ainsi le recours à la C.C.D.L.T.S. qui est une procédure plus lourde [13]. En comparant les équations (3c) et (4a), on obtient :

$$
\Delta V=(q / \varepsilon) \int_{0}^{w-\lambda} N_{\mathrm{t}}(x) \cdot x \cdot \mathrm{d} x .
$$

La largeur, $\lambda$ de la zone de transition est indispensable pour l'obtention de la concentration en pièges. Ce paramètre se calcule par intégration de l'équation de Poisson dans la région de transition, selon la même méthode que précédemment, mais avec une variable $X=x-(w-\lambda)$ :

$$
X \frac{q N(X)}{\varepsilon}=\frac{\mathrm{d}}{\mathrm{d} X}\left\{V-X \frac{\mathrm{d} V}{\mathrm{~d} X}\right\}
$$

soit

$$
\int_{0}^{\lambda} X \cdot[q N(X) / \varepsilon] \cdot \mathrm{d} X=V(w)-V(w-\lambda)
$$

on obtient donc,

$$
\begin{array}{rl}
{\left[E_{\mathrm{f}}-E_{\mathrm{t}}\right] / q=(q / \varepsilon) \int_{w-\lambda}^{w}} & N(x) \times \\
\times & {[x-(w-\lambda)] \cdot \mathrm{d} x}
\end{array}
$$

$E_{\mathrm{f}}$ et $E_{\mathrm{t}}$ représentent respectivement les niveaux de Fermi et des pièges monoénergétiques.

Cependant, l'utilisation de la relation (5) suppose la connaissance préalable de $E_{\mathrm{f}}-E_{\mathrm{t}}$ et donc, nécessite des mesures complémentaires (D.L.T.S. par exemple). Par ailleurs, la concentration $N(x)$, n'est pas toujours connue sur toute l'étendue, entre $w-\lambda$ et $w$, comme nous pouvons le constater sur la figure 5d. Pour surmonter ces difficultés, nous proposons une formulation analytique simple permettant de déterminer $\lambda$ à partir d'une valeur particulière $\lambda_{0}$ déduite de la mesure de capacité. Adoptons le modèle gaussien de pénétration des électrons libres dans la zone désertée ; leur concentration $n(x)$, peut s'exprimer sous la forme,

$$
\begin{array}{r}
n(x)=N(w) \cdot \exp \left[-(x-w)^{2} /\left(2 L_{\mathrm{D}}^{2}\right)\right] \\
\text { avec } x<w
\end{array}
$$

dans laquelle $L_{\mathrm{D}}$ correspond à la longueur de Debye :

$$
L_{\mathrm{D}}=\left[\varepsilon \cdot k \cdot T / N(w) \cdot q^{2}\right]^{1 / 2} .
$$

A l'équilibre thermodynamique, la «vitesse » d'émission $e_{\mathrm{n}}$ d'un niveau-piège à électrons, est liée à la «vitesse de capture » $c_{\mathrm{n}} \cdot n(x)$, selon l'équation (1). Mais, à l'intersection des niveaux $E_{\mathrm{t}}$ et $E_{\mathrm{f}}($ en $x=w-\lambda)$, la concentration en électrons libres étant $n(w-\lambda)$, ces deux «vitesses » sont égales :

$$
e_{\mathrm{n}}=n(w-\lambda) \cdot c_{\mathrm{n}} .
$$

Compte tenu de la relation (6), il en résulte :

$$
\begin{aligned}
n(w-\lambda)=e_{\mathrm{n}} / c_{\mathrm{n}}= & N(w) \times \\
& \times \exp \left[-\lambda^{2} /\left(2 L_{\mathrm{D}}^{2}\right)\right]=N_{\lambda}
\end{aligned}
$$

$N_{\lambda}$ est donc une constante caractéristique du niveau profond. Pour l'obtenir on considère la configuration particulière de la figure $2 \mathrm{~d}$, pour laquelle la largeur de la région désertée est égale à celle de la zone de transition $\lambda_{0}=\varepsilon \cdot A / C_{0} ; C_{0}$ représente la valeur mesurée de la capacité pour cette configuration et $A$ l'aire de la grille de la jonction. Nous en déduisons :

$$
N_{\lambda}=N\left(\lambda_{0}\right) \cdot \exp \left[-\lambda_{0}^{2} /\left(2 L_{\text {DO }}^{2}\right)\right]
$$

avec, d'après (3c), pour $w=\lambda_{0}$

$$
N\left(\lambda_{0}\right)=\left.\frac{\varepsilon}{q \cdot w} \frac{\mathrm{d} V}{\mathrm{~d} w}\right|_{w=\lambda 0}
$$

et $L_{\mathrm{DO}}$ donnée par la relation (7). 
Il est ainsi possible de connaître la constante $N_{\lambda}$. Compte tenu de la relation (9), on exprime $\lambda$, la largeur de la zone de transition par:

$$
\lambda(w)=\left\{\frac{2 \cdot \varepsilon \cdot k \cdot T}{q^{2} \cdot N(w)} \cdot \log \left(\frac{N(w)}{N_{\lambda}}\right)\right\}^{1 / 2} .
$$

Finalement, des relations ( $3 c$ ) et $(4 b)$, nous pouvons déduire :

- le profil des porteurs libres :

$$
N(w)=\frac{\varepsilon}{q \cdot w} \frac{\mathrm{d} V}{\mathrm{~d} w}
$$

- le profil des pièges :

$$
N_{\mathrm{t}}(w-\lambda)=\frac{\varepsilon}{q \cdot(w-\lambda)} \cdot \frac{\mathrm{d}(\Delta V)}{\mathrm{d}(w-\lambda)}
$$

la largeur de la zone désertée étant donnée par $w=\varepsilon \cdot A / C$ et $\lambda$, celle de la zone de transition par la relation (12).

Pour une meilleure précision et une plus grande fiabilité des résultats, nous avons procédé par acquisition et exploitation automatique des données à l'aide d'un micro-ordinateur TRS80. L'organigramme de la figure $3 a$ indique les principales étapes de calcul.

2.3 Domaine DE VAlidité. - Nous discutons dans ce paragraphe, des possibilités et limites de notre technique en déterminant les concentrations en porteurs libres et en centres profonds mesurables dans le cas du silicium monocristallin, et du domaine énergétique $E_{\mathrm{c}}-E_{\mathrm{t}}$ accessible, compte tenu du temps de réponse de l'appareillage.

Le capacimètre Boonton 72B utilisé, comporte une détection synchrone calée sur $1 \mathrm{MHz}$. En négligeant les résistances de contact et des circuits extérieurs, la capacité mesurée $C_{\mathrm{m}}$ est [16] :

$$
C_{\mathrm{m}}=C \cdot 2 \pi \cdot f /\left[1+\left(R_{\mathrm{s}} \cdot C \cdot 2 \pi \cdot f\right)^{2}\right]
$$

avec

$C$, la capacité de transition de la jonction ;

$f$, la fréquence de mesure $(f=1 \mathrm{MHz})$;

$R_{\mathrm{s}}$, la résistance de la zone neutre soit $R_{\mathrm{s}}=l /\left(q \cdot n \cdot \mu_{\mathrm{n}} \cdot A\right)$;

$l$ étant l'épaisseur de la zone neutre ;

$\mu_{\mathrm{n}}$, la mobilité des électrons libres dans Si $\left(\mu_{\mathrm{n}}=\right.$ $1300 \mathrm{~cm}^{2} / \mathrm{V}$. s à $300 \mathrm{~K}$ )

et $A$, l'aire de la grille de la diode.

$C_{\mathrm{m}} \quad$ ne représente la capacité de transition que si :

$$
\left(R_{\mathrm{s}} \cdot C \cdot 2 \pi \cdot f\right)^{2} \ll 1 .
$$

Cette inégalité fixe la concentration minimale en porteurs libres mesurable, compte tenu de la précision souhaitée, de l'épaisseur du substrat, et de la tempérautre. Nous avons résolu l'inéquation pour une valeur de capacité correspondant à la longueur de Debye $\left(C=\varepsilon \cdot A / L_{\mathrm{D}}\right)$, laquelle représente l'étendue minimale de la zone désertée.

Dans le cas d'un substrat de silicium à couche épitaxiale $(1=20 \mu \mathrm{m})$, pour une précision de $1 / 100$, à température ambiante, on obtient $n>10^{12} \mathrm{~cm}^{-3}$. La limite supérieure est fixée à environ $10^{17}$ $\mathrm{cm}^{-3}$ en raison de la faible extension de la charge d'espace qui en résulterait.

En ce qui concerne les centres profonds, le domaine de concentration accessible se situe entre les valeurs extrêmes suivantes :

i) La limite inférieure est déterminée par la sensibilité de détection de l'appareillage ; grâce à la possibilité de mesures différentielles moyennées, celle-ci atteint: $N_{\mathrm{t}} / N_{\mathrm{d}}=2 . \Delta C / C=10^{-5}$;

ii) La limite supérieure est fixée par la densité de dopants superficiels $N_{\mathrm{d}}$ par rapport à celle des centres compensateurs $N_{\mathrm{t}}$.

Enfin, la bande passante de la chaîne de mesure $\left(t_{0} \min =50 \mu \mathrm{s}\right)$ et la gamme de température autorisée par le cryostat (77 à $400 \mathrm{~K})$, permettent de mesurer la densité des pièges dont la profondeur énergétique s'étend du milieu de la bande interdite jusqu'à environ $120 \mathrm{meV}$ au-dessous de la limite de la bande de conduction.

La figure $3 \mathrm{~b}$ indique le domaine d'utilisation de cette méthode relativement aux principales grandeurs mesurées.

\section{Résultats expérimentaux et discussion.}

Afin de tester notre méthode de profilométrie, nous l'avons utilisée dans deux situations rencontrées très fréquemment dans la technologie des composants : i) cas de l'or thermiquement diffusé dans le silicium et ii) cas du phosphore introduit par implantation ionique dans le même matériau. Les structures étudiées ont été réalisées sur un substrat en silicium monocristallin épitaxié, de type $\mathbf{N}$ dopé au phosphore (résistivité $=8$ à $13 \Omega$-cm).

\subsection{ApPlication AUX STRUCTURES DifFusÉES. -} Des substrats de silicium ont été soumis à une diffusion d'or, à une température de $870{ }^{\circ} \mathrm{C}$ pendant $30 \mathrm{~min}$; les contacts Schottky ( $\mathrm{Au} / \mathrm{Si}$ ) ont été réalisés par dépôt sous vide sur une surface de 0,015 $\mathrm{cm}^{2}$. Les caractéristiques $C_{\mathrm{he}}\left(V_{\mathrm{r}}\right)$ et $C_{\mathrm{hf}}\left(V_{\mathrm{r}}\right)$ ont été acquises à $273 \mathrm{~K}$ en utilisant un temps $t_{0}$ de $100 \mu \mathrm{s}$.

Les résultats obtenus sont rapportés sur la figure 4. Ils confirment les propriétés connues de l'or dans le silicium de type $\mathrm{N}$ :

i) le caractère compensateur du centre profond qu'il introduit (la concentration en porteurs libres diminue par rapport au dopage initial) ;

ii) un profil quasi uniforme même pour des temps de diffusion courts. 

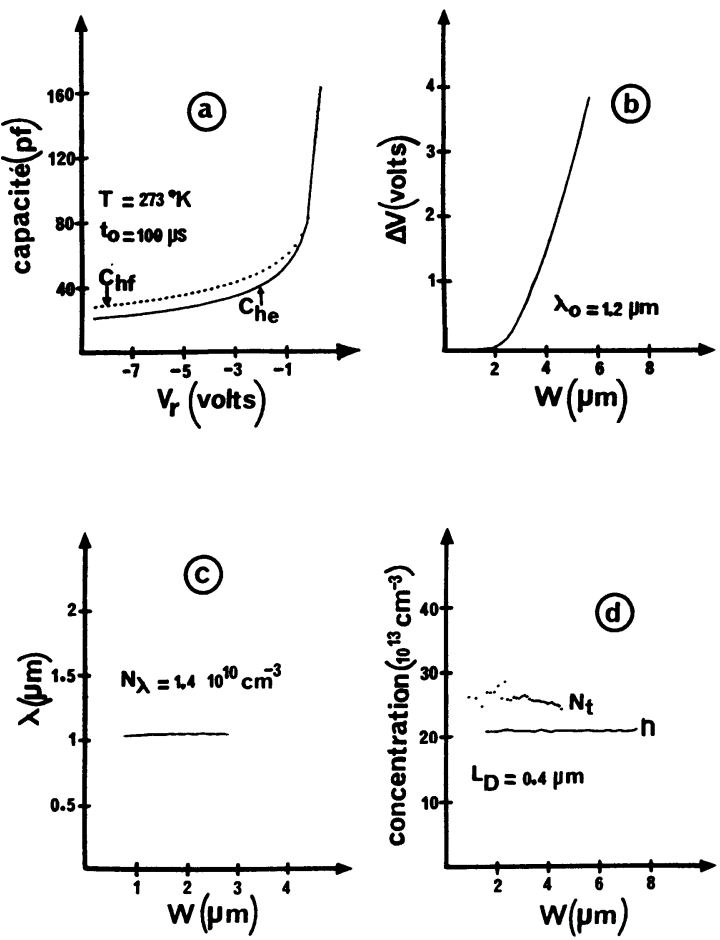

Fig. 4. - Résultats relatifs à une diode ayant subi une diffusion d'or et analysée à $273 \mathrm{~K}$ : a) caractéristiques $C_{\text {he }}\left(V_{\mathrm{r}}\right)$ et $\left.C_{\mathrm{hf}}\left(V_{\mathrm{r}}\right), \mathrm{b}\right)$ courbe $\Delta V(w)$, c) courbe $\lambda(w)$, d) profils des porteurs libres $(n(w))$ et des centres profonds $\left(N_{t}(w)\right)$.

[Gold diffused diode tested at $273 \mathrm{~K}$ : a) $C_{\mathrm{he}}\left(V_{\mathrm{r}}\right)$ and $C_{\mathrm{hf}}\left(V_{\mathrm{r}}\right)$ characteristics, b) $\Delta V(w)$ curve, c) $\lambda(w)$ curve, d) free carrier $(n(w))$ and deep trap $\left(N_{t}(w)\right)$ profiles.]

La position énergétique des pièges a été déterminée à partir de la constante $N_{\lambda}$; rappelons que celle-ci correspond à la concentration en porteurs libres, lorsque le niveau de Fermi est confondu avec le niveau-piège $E_{\mathrm{t}}: N_{\lambda}=N_{\mathrm{c}} \exp \left[\left(E_{\mathrm{t}}-E_{\mathrm{c}}\right) / k . T\right]$ avec $N_{\mathrm{c}}$ la densité effective d'états dans la bande de conduction. Nous avons ainsi obtenu pour $N_{\lambda}$ la valeur de $1,4 \times 10^{10} \mathrm{~cm}^{-3}$ : ce qui donne $E_{\mathrm{c}}-E_{\mathrm{t}}=$ $0,51 \mathrm{eV}$. Cette profondeur énergétique est en accord avec les valeurs indiquées dans la littérature [16].

Notons un résultat important quant à la validité de cette méthode : la somme des concentrations mesurées en centres profonds $\left(N_{\mathrm{t}}=27 \times 10^{13} \mathrm{~cm}^{-3}\right.$ en moyenne) et en porteurs libres $\left(n=20 \times 10^{13}\right.$ $\mathrm{cm}^{-3}$ ) est sensiblement égale au dopage initial calculé à partir de la résistivité du matériau de départ $\left(N_{\mathrm{d}}=2\right.$ à $\left.5 \times 10^{14} \mathrm{~cm}^{-3}\right)$.

\subsection{APPliCATION AUX STRUCTURES IMPLANTÉES.} - Une dose de $10^{12}$ atomes $/ \mathrm{cm}^{2}$ de phosphore a été introduite par implantation ionique dans des substrats à couche épitaxiale, identiques au précédent. Nous avons opéré à température ambiante avec une énergie de $180 \mathrm{keV}$. Des structures Schottky $(\mathrm{Au} / \mathrm{Si})$ ont été ensuite réalisées par métallisation sous vide sur une surface de $0,0035 \mathrm{~cm}^{2}$. La technique de profilométrie capacitive a été mise en œuvre à une température de $203 \mathrm{~K}$ en utilisant un temps d'échantillonnage $t_{0}$ de $100 \mu \mathrm{s}$.
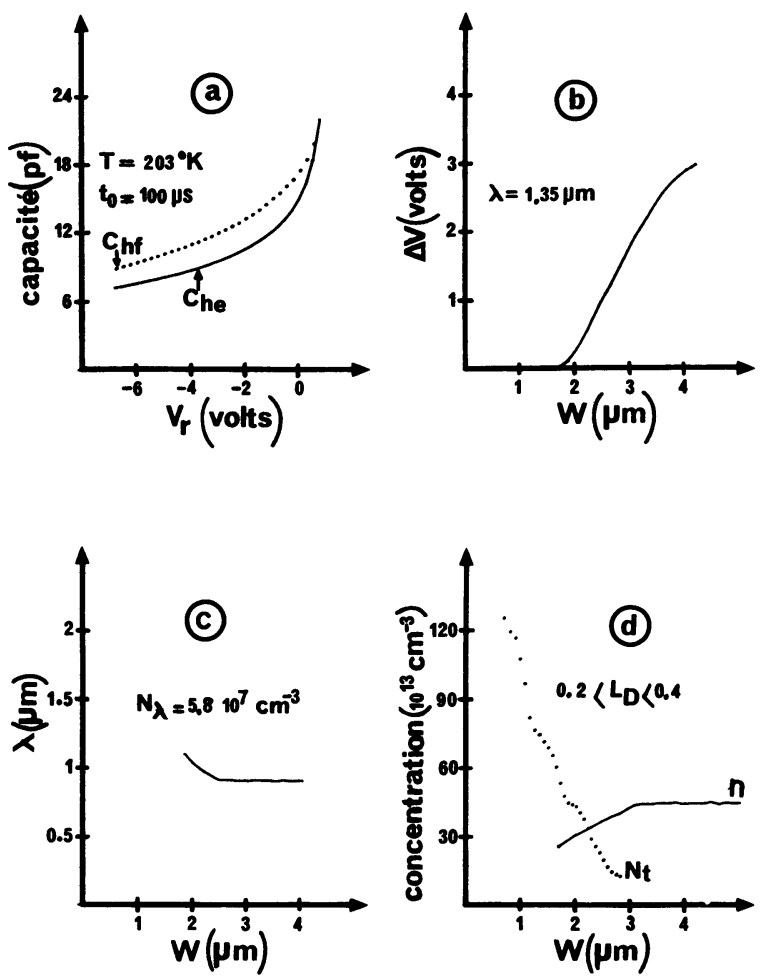

Fig. 5. - Résultats relatifs à une diode implantée au phosphore analysée à $203 \mathrm{~K}:$ a) caractéristiques $C_{\text {he }}\left(V_{\mathrm{r}}\right)$ et $C_{\mathrm{hf}}\left(V_{\mathrm{r}}\right)$, b) courbe $\Delta V(W)$, c) courbe $\lambda(W)$, d) profils des porteurs libres $(n(w))$ et des centres profonds $\left(N_{\mathrm{t}}(w)\right)$

[Phosphorus ion-implanted diode tested at $203 \mathrm{~K}$ : a) $C_{\mathrm{he}}$ and $C_{\mathrm{hf}}$ versus $V_{\mathrm{r}}$ curves, b) $\Delta V$ versus $W$ curve, c) $\lambda$ versus $W$ curve, d) free carrier $(n(w))$ and deep trap $\left(N_{\mathrm{t}}(w)\right)$ profiles.]

Les résultats expérimentaux obtenus sont représentés sur la figure 5 . Ils permettent de confirmer deux points :

i) le caractère compensateur du niveau P-V (association phosphore-oxygène) habituellement prépondérant dans ce type de traitement $[17,18]$;

ii) l'allure gaussienne du profil de ces défauts. La longueur de Debye $\left(0,2<L_{\mathrm{D}}<0,4 \mu \mathrm{m}\right)$ est ici supérieure à l'écart type théorique de la distribution des ions implantés $(0,04 \mu \mathrm{m})$; l'incertitude sur le profil mesuré est, par suite, très importante ; seule l'allure est prise en considération.

Nos résultats expérimentaux montrent que la mesure de la concentration des porteurs libres et des centres profonds s'effectue dans des zones quasi disjointes lorsque le matériau est très compensé (Fig. 5d) ; la concentration en porteurs libres 
demeure par suite inconnue dans la région où l'on connaît celle des pièges : ceci est une difficulté supplémentaire en ce qui concerne la mesure des coefficients de capture par capacité ou courant transitoires [19].

\section{Conclusion.}

La caractéristique $C_{\mathrm{he}}(V)$ présente un double intérêt :

i) L'évolution de la charge d'espace est contrôlée uniquement par les porteurs libres. Leur profil est, par suite, obtenu indépendamment de la présence des pièges.

ii) La zone prospectée est augmentée grâce à la réduction de la charge d'espace consécutive au « gel » des pièges dans l'état « occupé ».

Le calcul de la largeur de la zone de transition $\lambda$ à partir de la pénétration gaussienne des électrons libres dans la zone désertée s'avère bien adapté. Le profil des centres profonds est obtenu sans recours à des connaissances préalables comme leur position énergétique dans la bande interdite.

Cette méthode demeure, cependant, limitée dans son principe par :

i) la faible étendue de la zone prospectée à cause du champ électrique maximum admissible dans le semiconducteur ;

ii) la médiocrité de la résolution spatiale dans le cas de matériaux très compensés.

Les résultats obtenus pour l'or diffusé dans le silicium et le phosphore implanté dans le même matériau sont en accord avec les connaissances actuelles sur les effets induits par ces traitements. Nous avons mis en évidence l'importance d'une détermination précise du profil des porteurs libres quant à la mesure des coefficients de capture. La caractéristique $C_{\text {he }}(V)$ apparaît donc comme un outil précieux pour la caractérisation des semiconducteurs.

\section{Bibliographie}

[1] Colloquium on dopant and carrier concentration in semiconductors layers, IEEE Trans. Electron Devices digest $\mathbf{N 5}$ (1984).

[2] Kennedy, D. P., Murley, P. C., Kleinfelder, W., IBM J. Res. Dev. 12 (1968) 399-409.

[3] Miller, G. L., IEEE Trans. Electron Devices, ED 19 (1972) 1103-1108.

[4] LANG, D. V., J. Appl. Phys. 45 (1974) 3023.

[5] SAH, C. T., REDDI, V. G. K., IEEE Trans Electron Devices ED 11 (1964) 245-349.

[6] ZонтA, Y., Solid-State Electron. 16 (1973) 10291035.

[7] Gото, G., Yanagosawa, S., Wada, O., Takanashi, H., Appl. Phys. Lett. 23 (1973) 150-151.

[8] Lefevre, H., Schultz, M., Appl. Phys. 53 (1977) 45-53.

[9] Kimmerling, L. C., J. Appl. Phys. 45 (1974) 18391845.

[10] Loualiche, S., Nouailhat, A., Guillot, G., Solid State Electron. 25 (1982) 577-582.
[11] Goto, G., Yanagisawa, S., Wada, O., TaKaNASHI, H., Jpn J. Appl. Phys. 13 (1974) 11271133.

[12] Johnson, N. M., BARTElink, D. J., Gold, R. B., Gibbons, J. F., J. Appl. Phys. 50 (1979) 48284833.

[13] LI, M. F., SAH, C. T., IEEE Trans. Electron Devices ED 29 (1982) 306-315.

[14] Remaki, B., Thèse Doctorat, L.P.M.-INSA de Lyon (1985).

[15] Brotherton, S. D., Solid State Electron. 19 (1976) 341-342.

[16] Wiley, J. D., Miller, G. L., IEEE Trans. Electron Devices ED 22 (1975) 265-272.

[17] Lang, D. V., Grimmeiss, H. G., MeiJer, E., JARos, M., Phys. Rev. B 22 (1980) 3917.

[18] Troxell, J. R., Solid State Electron. 26 (1983) 539548.

[19] Balland, B., Remaki, B., Pinard, P., Mercier, A., J. Physique colloq. 44 (1983) C5-319-324.

[20] Pons, D., J. Appl. Phys. 55 (1984) 3644-3657. 